Chronic Obstructive Pulmonary Diseases:

Journal of the COPD Foundation

COPD

FOUNDATION

\author{
Letter to the Editor
}

\title{
Progressing the Progress Note for COPD
}

\author{
Deborah A. Goss, MD $^{1}$
}

\begin{abstract}
Abbreviations: electronic medical record, EMR; subjective-objective-assessment-plan, SOAP; goal-oriented scientific survey, GOSS; chronic obstructive pulmonary disease, COPD; Global initiative for chronic Obstructive Lung Disease, GOLD; COPD Assessment Test, CAT; pulmonary function tests, PFTs; forced expiratory volume in 1 second, FEV $\mathbf{1}$; 6-Minute Walk Test, 6MWT; cardiopulmonary stress test, CPST; computed tomography, CT; short-acting beta2-agonists, SABA; 4 times per day, qid; use when necessary, PRN; phosphodiesterase, PDE; bronchoalveolar lavage, BAL; emergency department, ED; percutaneous endoscopic gastrostomy, PEG; next of kin, NOK

Citation: Goss DA. Progressing the progress note for COPD. Chronic Obstr Pulm Dis. 2017;4(1):4-6. doi: https://doi.org/10.15326/ jcopdf.4.1.2016.0155
\end{abstract}

1 Hackensack University Medical Center, Hackensack, New Jersey

\section{Address correspondence to:}

Deborah A. Goss, MD

Email: gossda0325@gmail.com

\section{Keywords:}

chronic obstructive pulmonary disease; COPD; subjectiveobjective-assessment plan; SOAP; electronic medical record; goaloriented scientific survey

\section{Dear Editor:}

Electronic medical records (EMRs) can either improve medical care or impede it (i.e., "garbage in equals garbage out"). The ability to take a vast amount of material and methodically and meticulously extract the essential information is a skill for which medical documentation is long overdue. Since the development of the subjective-objective-assessment-plan (SOAP) note in the 1960's by Lawrence Weed, MD, little has changed in the way physicians write a progress note. ${ }^{1}$ While EMRs dramatically improved the access and legibility of physicians' notes, it also compounded the problem by giving physicians an easy way to overpopulate their notes with bulky and wordy results of diagnostic testing. ${ }^{2}$ By the same token, excluding this information leaves the reader to the unhappy task of reviewing endless PDF files on their own.

In response to this need, I propose an overhaul to the assessment/plan portion of the SOAP note. By adding a goal-oriented scientific survey (GOSS or GO-SOAP) of the patient, this will not only accurately describe a patient's condition and disease state, but also integrate evidence-based medicine into the everyday practice of medicine. This adds an artificial intelligence to an otherwise historical record.

For example, the following is a SOAP note assessment and plan with a goal-oriented scientific survey (GOSS note) for chronic obstructive pulmonary disease (COPD):

\section{SOAP Assessment/Plan:}

COPD- Patient has increased shortness of breath today. Will increase bronchodilators and repeat chest X-ray. Will give empiric trial of steroids and antibiotics for acute exacerbation. Patient to follow-up in 1 week.

\section{GOSS Assessment/Plan: ${ }^{a}$}

COPD, Gold 2B. ${ }^{3}$

Tobacco: 40 pack years. Off cigarettes. Continue smoking cessation.

CAT score ${ }^{4}$ (Dec 2015): 12

PFTs (Dec 2015): FEV 1.77 L, 78\%. FEV 1 decline $<40 \mathrm{~mL}$ per year.

6MWT (Dec 2015): 412 meters, without desaturation

CPST (Oct 2014): $\mathrm{VO}_{2} \max 12 \mathrm{ml} / \mathrm{kg} / \mathrm{min}$. Patient enrolled in Pulmonary Rehab.

CT chest (Feb 2015): RLL nodule, enrolled in lung cancer screening program

Alpha-1 level (Sept 2013): normal, MM

Vaccines: She has had a pneumonia vaccine 
(2013) and will need booster age 70. She has had a pertussis (2012) and influenza vaccine.

Failed: Inhaled steroids- paradoxical bronchospasm Treatment: Now with acute exacerbation, will give antibiotics and oral steroids. Increase SABA to qid and prn. Will consider a trial of PDE inhibitors as patient has had repeat exacerbations this year. Will be due to follow-up PFTs in Dec 2016.

\section{Exacerbations:}

Oct 2012 Hospitalized with antibiotics and steroids Jan 2015 BAL with staphylococcus, treated with antibiotics

Mar 2015 ED treated with antibiotics and steroids

Advanced directives: Full Code, agrees to trach/ PEG if needed. NOK is Husband with cell phone 999-999-9999;

GOLD=Global initiative for chronic Obstructive Lung Disease; CAT $=$ COPD Assessment Test; $\mathrm{PFTs}=$ pulmonary function tests; $\mathrm{FEV}_{1}=$ forced expiratory volume in 1 second; 6MWTs=6-Minute Walk Test; $\mathrm{CPST}=$ cardiopulmonary stress test; $\mathrm{CT}=$ computed tomography; SABA=short-acting beta2-agonists; qid=4 times per day; prn=use when necessary; $\mathrm{PDE}=$ phosphodiesterase; $\mathrm{BAL}=$ bronchoalveolar lavage; $\mathrm{ED}=$ emergency department; $\mathrm{PEG}=$ percutaneous endoscopic gastrostomy; NOK=next of kin

a please note: patent pending

In this note, the patient has met the goal for smoking cessation, but may not be at goal for the number of exacerbations per year. The objective evaluation is using the pulmonary function testing and Global initiative for chronic Obstructive Lung Disease (GOLD) classification to decide on medications and the enrollment into pulmonary rehabilitation. The subjective survey is the inclusion of the CAT score and/ or other validated surveys to quantify patient symptoms instead of self-reporting. In conclusion, while this form of documentation in the assessment/plan note section may initially seem cumbersome, it can greatly reduce the need to rummage through a large stack of electronic and paper records. Overall, this will reduce the time needed to evaluate and manage each patient. It serves to allow for quick comparison to prior visits, reminders for necessary screenings and follow-up evaluations, keeping track of the past successes and failures in management, as well as allowing for new avenues which have not yet been explored. However, the beauty is in the eye of the beholder. Physicians must decide for themselves what they feel would best belong in their own note. This is just a simple proposal to show a glimpse of the possibilities for a more interesting and useful medical record.

\section{Acknowledgements}

Special thanks to Barbara Reich, our outstanding medical librarian, for assistance with this manuscript and numerous literature searches to better the care of our more interesting and challenging patients. 


\section{References}

1. Jacobs L. Interview with Lawrence Weed, MD-The father of the problem-oriented medical record looks ahead. Perm J. 2009;13 (3):84-89. doi: https://doi.org/10.7812/TPP/09-068

2. Palma G. Electronic Health Records: The Good, the Bad and the Ugly. Beckers Health IT \& CIO Review website. http:// www.beckershospitalreview.com/healthcare-informationtechnology/electronic-health-records-the-good-the-bad-andthe-ugly.html Published October 14, 2014. Accessed August 2016.

3. Global initiative for chronic Obstructive Lung Disease (GOLD). Global Strategy for the Diagnosis, Management and Prevention of COPD, 2015. GOLD website. http://goldcopd.org/ Published 2015. Accessed August 2016.

4. Jones PW, Harding G, Berry P, Wiklund I, Chen W-H, Leidy NK. Development and first validation of the COPD Assessment Test. Eur Respir J. 2009; 34: 648-654.

doi: https://doi.org/10.1183/09031936.00102509 